may present with stridor, feeding difficulties, symptoms of inhalation, and respiratory distress. ${ }^{1}$ Some arise from thyroglossal remnants and some are simple mucus retention cysts. Thyroglossal cysts at the upper end of the developmental tract occur in the region of the foramen caecum. Mucus retention cysts typically occur in the groove between the tongue and epiglottis; the cysts reported here were probably of this last kind.

Although these cysts are rare, they can be easily diagnosed. The technique of inserting the little finger along the surface of the tongue has been recommended in the routine examination of children with chronic or recurrent stridor, ${ }^{1}$ although it is not mentioned in standard paediatric textbooks. Palpation of a tense cyst by the little finger produces an unmistakable sensation. We have shown the usefulness of this technique, although, as case 3 illustrates, palpation must extend to the epiglottis to avoid missing the cyst.

We encountered several perioperative problems. They included airways obstruction during induction of anaesthesia and postoperatively, and difficulties in finding the roof of the cyst once the contents had been aspirated. We therefore propose the following operative method: an anaesthetic that can be inhaled should be given, and a traction stitch placed in the tongue. Pulling the tongue forward should relieve upper airway obstruction. Once the child is paralysed a holding stitch should be inserted into the cyst roof to allow the contents to drain and facilitate endotracheal intubation and simple marsupialisation. Postoperatively the tongue stitch should remain taped to the face until all stridor has resolved. This may avoid tracheostomy with its potential for serious complications in this age group. ${ }^{23}$ Preoperative tracheostomy with local anaesthesia should be considered in hospitals without an experienced paediatric anaesthetist. A vertical rather than a transverse incision is preferable for urgent tracheostomies because deviation from the midline is less likely to occur. ${ }^{23}$ The cosmetic result is quite acceptable.

The occurrence of four cases of glossal cysts in a nine month period in a population of about 300000 may be a coincidence. Glossal cysts have not previously been reported from Papua New Guinea and paediatricians and surgeons with considerable experience in the country have not seen a case (JKA Clezy et al, personal communication), but previous cases could have been misdiagnosed as pneumonia, which is a common cause of death in this community.

The authors acknowledge the help of Dr F Shann and Professor PD Phelan in preparing this manuscript.

\footnotetext{
References

1 Phelan PD, Landau LI, Olinsky A. Respiratory illness in children. 2nd ed. Oxford: Blackwell Scientific Publications, 1982:123.

2 Perotta RJ, Schley WS. Paediatric tracheostomy: a 5 yearo comparison study. Arch Otolaryngol 1978;104:318-21.

3 Gilmore BB, Michelson JA. Paediatric tracheotomy controversies in management. Otolaryngol Clin North Am 1986;19:141-51
}

Correspondence to Dr MD Harari, Intensive Care Unit, Royal Children's Hospital, Flemington Road, Parkville, Victoria, Australia 3052

Received 10 April 1987

\title{
Cellular phosphate in renal tubular acidosis
}

\author{
A CHALLA, G VRIONIS, AND P D LAPATSANIS
}

Department of Paediatrics, University of Ioannina Medical School, Ioannina, Greece

SUMMARY In two infants with distal renal tubular acidosis phosphate depletion was observed in the extracellular and intracellular compartments of the erythrocytes. Treatment corrected this disturbance over a period of several months. Cell phosphate deficiency may contribute to the adverse effects of renal tubular acidosis on bone.
Distal renal tubular acidosis is characterised by low serum concentrations of bicarbonate, raised serum concentrations of chloride, sustained metabolic acidosis and inadequate urinary acidification $(\mathrm{pH}>6)$. In addition to hyperchloraemic acidosis, there is often a variety of associated disturbances in electrolyte metabolism like hypokalaemia and hypercalciuria. The clinical manifestations of these abnormalities may include muscle weakness, osteomalacia or 
rickets, growth retardation, and nephrocalcinosis.

This study follows the metabolic changes of phosphate in and out of the red cells, at both the acidotic stage and during treatment.

\section{Patients and methods}

Two preterm infants (small for dates) with gestational ages of 34 and 32 weeks and birth weights of 1100 and $1130 \mathrm{~g}$, respectively, were studied. Both neonates had previously been treated for late metabolic acidosis. The recurrence of acidosis in both infants at nine and 12 weeks after birth led to the diagnosis of hyperchloraemic acidosis and sodium bicarbonate was readministered.

We measured serum calcium concentration by titration against edetic acid (Calcette model 4008, Precision System Inc, Massachusetts, United States). Alkaline phosphatase activity, adenosine triphosphate, and 2, 3 diphosphoglycerate concentrations were measured photometrically with kits from Boehringer and Sigma. Inorganic phosphate concentration in plasma and red cells was also measured photometrically by the method of Challa et al. ${ }^{1}$ Serum concentration of potassium was measured by flame photometry, serum bicarbonate with a pH blood gas analyser (Acid Base Laboratory, Radiometer, Copenhagen), and serum chloride with the photometric method of Zall et al. ${ }^{2}$

\section{Results}

The figure shows that the bicarbonate concentrations were low at the time of diagnosis ( 6 and $15 \cdot 5$ $\mathrm{mmol} / \mathrm{l}$, respectively) and took about two months of treatment to be corrected. The chloride concentrations took far longer to be corrected and even after a year's treatment the values in both infants were in the upper limit of normal.

Serum concentration of calcium was low before treatment $(2.13$ and $1.78 \mathrm{mmol} / \mathrm{l}$, respectively); administration of bicarbonate and $0.3 \mu \mathrm{g} / \mathrm{d} 1 \alpha$ hydroxyvitamin $\mathrm{D}_{3}\left(1 \alpha \mathrm{OHD}_{3}\right)$ in the first week of treatment increased it. The dose of $1 \alpha \mathrm{OHD}_{3}$ was gradually lowered to a maintenance dose of 0.05 $\mu \mathrm{g} / \mathrm{d}$. Plasma concentrations of inorganic phosphate were also low at diagnosis $(1.43$ and $1.03 \mathrm{mmol} / \mathrm{l}$, respectively) and were corrected slowly over about two months. The erythrocytes of these infants were found to be depleted of inorganic phosphate, with concentrations at the time of diagnosis of 0.26 and $0.43 \mathrm{mmol} / \mathrm{l}$ (normal range $0.80-1.13 \mathrm{mmol} / \mathrm{l}$ ). After administration of alkaline solutions they started to recover slowly, taking less than two months in the first case and over six months in the second.

Alkaline phosphatase activities were high at

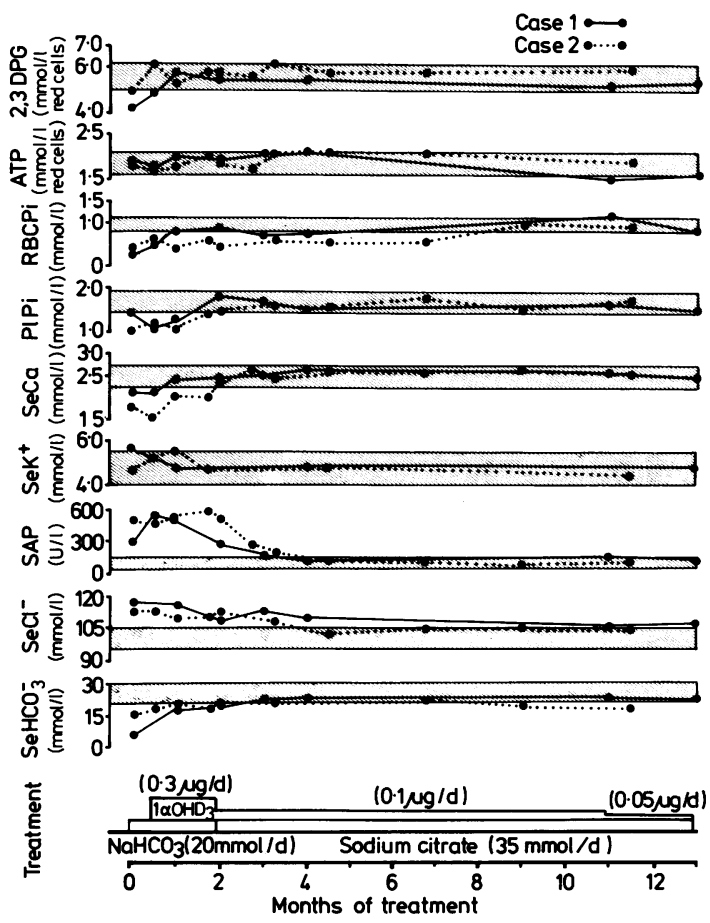

Figure Concentrations of bicarbonate $\left(\mathrm{HCO}_{3}^{-}\right)$, chloride $\left(\mathrm{Cl}^{-}\right)$, alkaline phosphatase (SAP), potassium $\left(\mathrm{K}^{+}\right)$and calcium $(\mathrm{Ca})$ in serum, inorganic phosphate in plasma, and red cells (PIPi and RBCPi) and red cell ATP and 2,3 DPG of two cases with renal tubular acidosis before and during treatment.

diagnosis in both cases but became normal within four months of treatment. The concentration of organic phosphate from adenosine triphosphate (ATP) in red blood cells was normal at the time of the diagnosis, when the cells were depleted of phosphate and did not change all through the study. Concentrations of 2,3 diphosphoglycerate were low normal or low at diagnosis and increased after the administration of bicarbonate.

\section{Discussion}

Although it is well established that acidosis may result in the liberation of phosphorus from organic compounds within cells, this hyperphosphataemia has been described in association with lactic acidosis. Other data suggest that ammonium chloride induced acidosis is not associated with increases in plasma phosphorus. ${ }^{3}$

In the two infants we studied with renal tubular 
acidosis phosphate depletion was found both extracellularly and inside the red blood cells; depletion may have been found in other cells as well. Preston et al reported depletion in red blood cells, leucocytes, and platelets in an adult case of proximal renal tubular acidosis. ${ }^{4}$ The intracellular changes in the case and the two we studied were more extensive than the extracellular ones and there was a time lag for repletion between the two compartments after treatment. Plasma inorganic phosphate returned to normal long before the chloride ion concentrations and the alkali reserves approached normal limits. In contrast, intracellular inorganic phosphate started repleting at a lower rate and in the second infant took up to six months before it was in the normal range. It is worth noting that this infant was diagnosed at a later stage ( 3 months) when the alkaline phosphatase was very high (500 IU/l; normal range 35-100) and remained high for a longer period than in the first child.

Despite the intracellular and extracellular inorganic phosphate depletion the ATP concentrations in the erythrocytes of the two cases did not show any significant changes and were within the normal limits. These findings were at variance with those of Preston et al, who found an initial decrease in ATP that was corrected after a month of treatment ${ }^{4} \mathrm{He}$ attributed this fall to intracellular acidosis due to potassium ion losses that had been exchanged with hydrogen ions. Acidosis inhibits glycolysis and consequently ATP production. In the two infants that we studied the plasma concentrations of potassium changed but were within the normal range. Another reason for these results might be that their study was of a chronic case of renal tubular acidosis in an adult who had also developed osteomalacia and muscle weakness.

Changes in $\mathrm{pH}$ also affect the concentrations of 2,3 diphosphoglycerate because acidosis leads to a fall and alkalosis to a rise of this metabolite. In our two cases the concentrations of 2,3 diphosphoglycerate were low normal or low at diagnosis and became normal within a month of treatment. In the patient described by Preston et al the recovery of the concentration of 2,3 diphosphoglycerate took far longer ( $>$ four months).

Serum concentration of calcium was abnormally low in the second infant and low normal in the first but recovered after several weeks of treatment. As has been reported in the past increased rates of acid production are accompanied by positive acid balances, increased urinary excretion rates, and nega- tive calcium balances. The urinary losses of calcium that accompany increased rates of fixed acid production are the consequence of reduced net renal tubular calcium reabsorption that seems to be independent of parathyroid hormone and vitamin D. ${ }^{5}$

The mechanisms by which augmented fixed acid production rates stimulate net bone resorption are not yet fully defined as the activation of the parathyroid hormone/vitamin D endocrine system does not mediate the effect of acidosis. Bone resorption must be either stimulated by direct effect of acidosis or as a consequence of some as yet undefined humoral mechanism. ${ }^{6}$

Although no radiological findings of rickets were evident, the alkaline phosphatase activities were abnormally high in both infants but started to become normal after treatment. Phosphate depletion in cells might be one factor for disturbing bone metabolism, which is reflected in the high activities reported.

From a clinical point of view late metabolic acidosis in small birth weight neonates should always be investigated for the pathological cause of origin.

\section{References}

${ }^{1}$ Challa A, Bevington A, Angier CM, Asbury AJ, Preston CJ Russell RGG. A technique for the measurement of orthophosphate in human erythrocytes and some studies of its determinants. Clin Sci 1985;69:429-34.

2 Zall DM, Fisher D, Garner MQ. Photometric determination of chlorides in water. Anal Chem 1956;28:1665-8.

3 Oster JR, Perez GO, Vaamonde CA. Relationship between blood $\mathrm{pH}$ and potassium and phosphorus during acute metabolic acidosis. Am J Physiol 1978;235:F345-51.

4 Preston CJ, Noorwali A, Challa A, et al. Intracellular inorganic phosphate and ATP levels in human blood erythrocytes, leucocytes and platelets in normal subjects and in diseases associated with altered phosphate metabolism. In: Massry SG, Letterie JM, Ritz E, eds. Advances in experimental medicine and biology vol. 151. New York and London: Plenum Press, 1982:147-55.

5 Weber HP, Gray RW, Dominguez JH, Lemann J Jr. The lack of effect of chronic metabolic acidosis on 25-OH-vitamin D metabolism and serum parathyroid hormone in humans. J Clin Endocrinol Metab 1976;43:1047-55.

6 Bushinsky DA, Krieger NS, Geisser DI, Grossman EB, Coe FL. Effects of $\mathrm{pH}$ on bone calcium photonfluxes in vitro. Am J Physiol 1983;245:F204-9.

Correspondence to Professor PD Lapatsanis, Department of Paediatrics, Medical School, University of Ioannina, Ioannina, Greece 45444.

Received 21 April 1987 